\title{
Síndrome nefrótico cortico-resistente secundario a mutación genética, a propósito de 2 casos clínicos
}

\author{
NEDIER PEDRAZA G. ${ }^{1}$, MARÍA LUISA CEBALLOS O. ${ }^{2}$, FRANCISCO CANO SCH. ${ }^{3}$ \\ 1. Becada del Programa de Especialista en Nefrología Infantil, Escuela de Postgrado, Facultad de Medicina, \\ Universidad de Chile. \\ 2. Becada del Programa de Especialista en Pediatría, Escuela de Postgrado, Facultad de Medicina, Universidad de Chile, \\ Hospital Luis Calvo Mackenna. \\ 3. Nefrólogo Pediatra, Hospital Luis Calvo Mackenna, Facultad de Medicina, Universidad de Chile.
}

\begin{abstract}
Corticoresistant Nephrotic Syndrome secondary to genetic mutation, 2 Case-report

Background: Around 10-30\% patients with Corticoresistant Nephrotic Syndrome (CRNS) have hereditary glomerulopathy. Objective: Describe 2 children with CRNS on chronic peritoneal dyalisis (CPD), with positive Podocin mutation genetic study. Case-report 1: A 4 years-old male with CRNS and diagnosis of focal segmental glomerulosclerosis (FSGS) at renal biopsy, with positive R 229Q and A 284V Podocin mutation genetic study. The treatment included Enalapril, steroids and Cyclophosphamide without remission, requiring CPD at 12 years-old. Case-report 2: A 6 years-old female with CRNS and diagnosis of focal segmental glomerulosclerosis (FSGS) at renal biopsy, with positive R 229Q and A 284V Podocin mutation genetic study. The treatment included Enalapril, steroids and Cyclophosphamide without remission, requiring CPD at 7 years-old. Conclusion: The genetic mutation study should be included in all CRNS cases, in order to guide the therapy and prognosis of the disease.

(Key words: Corticoresistant Nephrotic Syndrome, podocin, $4 \alpha$-actinin, A284V mutation, R229Q mutation) Rev Chil Pediatr 2008; 79 (4): 398-403
\end{abstract}

\section{RESUMEN}

En pacientes portadores de Síndrome Nefrótico Córtico Resistente (SNCR) se ha demostrado que entre el 10 y 30\% presentan glomerulopatías hereditarias. Objetivo: Describir dos niños portadores de SNCR en diálisis peritoneal crónica (DPC), cuyo estudio genético para mutación de podocina fue positivo. Caso 1: Paciente de sexo masculino, debuta a los 4 años con un SNCR, biopsia renal informa una Glomerulo Esclerosis Focal Segmentaria (GEFS), se maneja con enalapril, esteroides y ciclofosfamida, sin lograr 2008, aceptado para publicación el 10 de junio de 2008.

Correspondencia a:

Nedier Pedraza G.

E-mail: nedierpedraza@hotmail.com 
remisión. A los 12 años ingresa a DPC, estudio genético resulta positivo para mutación del gen de la podocina en los alelos R 229Q y A 284V. Caso 2: Paciente de sexo femenino, a los 6 años de edad debuta con un SNCR, biopsia renal informa GEFS, recibe tratamiento clásico sin respuesta, a los 7 años inicia DPC. Su estudio reporta mutacion de podocina alelos R229Q y A284V. Conclusión: El estudio genético debería ser incorporado en el estudio etiopatogénico de todo SNCR para orientar el tratamiento y pronóstico de la enfermedad.

(Palabras clave: Síndrome Nefrótico, Córtico resistente, nefrina, podocina, $\alpha$-actinina 4, mutación A284V, mutación R229Q)

Rev Chil Pediatr 2008; 79 (4): 398-403

\section{Introducción}

El síndrome nefrótico ( $\mathrm{SN})$ es una de las patologías nefrológicas más frecuentes en pediatría, y se caracteriza por edema generalizado, oliguria, proteinuria masiva, hipoalbuminemia e hipercolesterolemia ${ }^{1,2}$. La incidencia comunicada en población anglosajona es de 1-7 casos por 100 mil habitantes menores de 16 años, siendo mayor en poblaciones asiáticas y afroamericanas $^{7,8}$ y en países latinoamericanos como Colombia se ha comunicado una cifra de 2-7 casos por 100 mil menores de 18 años ${ }^{9}$.

Este síndrome puede ser primario o idiopático, o secundario a un amplio espectro de enfermedades que van desde la nefrosis congénita en el $\mathrm{RN}$, hasta las glomerulopatías propias del adulto. Puede igualmente ser secundario a enfermedades sistémicas como el lupus o una vasculitis, a un trastorno metabólico, o medicamen$\operatorname{tos}^{3-6}$.

La forma más frecuente de $\mathrm{SN}$ en pediatría es la Enfermedad por Cambios Mínimos (ECM) o Nefrosis Lipoidea, con un patrón histológico de fusión pedicelar sin otras alteraciones glomerulares, y en segundo lugar, la Glomeruloesclerosis Focal y Segmentaria (GEFS), sin embargo, existen comunicaciones que sugieren que la GEFS ha ido en creciente aumento en los últimos años en población pediátrica ${ }^{2}$. En el debut del $\mathrm{SN}$ no es posible distinguir entre ambas entidades, sin embargo, la respuesta al tratamiento con esteroides es distinta, con un $95 \%$ de los niños con ECM obteniendo remisión completa con prednisona, versus sólo el 30$40 \%$ de los pacientes con GEFS, porcentaje que en el tiempo va disminuyendo en relación al número de recaídas ${ }^{10,11}$. El esquema de tratamiento ha sido publicado previamente por la Rama de Nefrología de la Sociedad Chilena de Pediatría $^{12}$.

Con los avances en el estudio genético, hoy se sabe que entre el 10 y el $30 \%$ de los pacientes con SN corticoresistente presentan glomerulopatías hereditarias, como el síndrome congénito fines, la esclerosis mesangial difusa, GEFS familiar, síndrome de Denys Drash, etc. En la actualidad, en el enfoque inicial del estudio de todo paciente portador de un SNCR, debe plantearse el estudio genético, ya que en los casos en que se identifica una mutación positiva para un gen, debe evaluarse la real utilidad del tratamiento esteroidal y drogas inmunosupresoras como la ciclosporina o tacrolimus, con potenciales efectos iatrogénicos ${ }^{13,14}$.

Existen actualmente descritas varias mutaciones que se relacionan a SNCR afectando la expresión de proteínas de la barrera glomerular, como son la nefrina para el gen NPHS1, la podocina para el gen NPHS2, $\alpha$-actinina 4 para el gen ACTN4, entre otras ${ }^{15}$. En nuestro país no se han comunicado hasta el momento casos de SNCR de origen genético.

\section{Objetivo}

Descripción de dos pacientes pediátricos con síndrome nefrótico córtico resistente en los cuales se confirmó un origen genético secundario a una mutación para podocina.

\section{Caso clínico 1}

Escolar de sexo masculino, de 13 años de 
edad, sin antecedentes mórbidos durante el período de recién nacido ni primera infancia, cuya hermana mayor fue sometida a trasplante renal por insuficiencia renal crónica (IRC) secundaria a un $\mathrm{SN}$ de etiología no precisada.

Consulta por primera vez a los 3 años 11 meses por presentar disuria. En el servicio de urgencia se constató hematuria microscópica, creatinina plasmática de $0,33 \mathrm{mg} / \mathrm{dl}$ y ecografía renal que fue informada normal. Continúa en seguimiento, evoluciona posteriormente con persistencia de la hematuria y aparición de proteinuria y compromiso de la función renal en forma progresiva. A los 4 años se realizó ecografía renal que reporta nefromegalia bilateral.

A los 5 años de edad se efectuó biopsia renal, cuyo informe histológico fue compatible con cambios glomerulares mínimos, con leve ensanchamiento mesangial. La inmunofluorescencia resultó negativa y la microscopia electrónica confirmó una glomeruloesclerosis focal segmentaria.

Se realizó tratamiento con enalapril, esteroides y un ciclo de ciclosfosfamida, sin remisión de la enfermedad la cual progresó con compromiso de la función renal. A los 12 años la creatinina plasmática era de $4,5 \mathrm{mg} / \mathrm{dl}$ con una depuración de creatinina de $16 \mathrm{ml} / \mathrm{min}$, por lo cual ingresó al programa de diálisis peritoneal crónica. Se realizó estudio genético en busca de mutaciones en el gen de la podocina, cuyo análisis se efectuó en el Laboratorio de Biología Molecular del Hospital Clínico de la Universidad Católica de Chile, a partir de una muestra de sangre total fijada con EDTA.

Tras la centrifugación de la muestra, se procede a la extracción del DNA mediante kits comerciales o en forma manual, consiguiendo concentraciones de $40 \mu \mathrm{g} / \mu \mathrm{l}$, para luego amplificar el DNA por medio de reacción de polimerasa en cadena (RPC). Posteriormente con electroforesis en gel de poliacrilamina, se extraen los exones 5 y 7 y la muestra se digiere con las enzimas de restricción clai para el exón 5 y Hyn6 para el exón 7, con el fin de determinar mediante el patrón de bandeo de los alelos, las mutaciones R229Q y A284V, respectivamente.

En este paciente ambas mutaciones A284V y R229Q, resultaron positivas.

\section{Caso clínico 2}

Escolar de 9 años, sexo femenino, sin antecedentes mórbidos durante el período de recién nacido ni primera infancia, sin antecedentes familiares, que debutó a los 6 años de edad con neumonía por Mycoplasma pneumoniae, requiriendo hospitalización en Unidad de Tratamiento Intensivo. En su evolución se pesquisó hipoalbuminemia, hipercolesterolemia y proteinuria. Evolucionó con hipertensión arterial y oliguria, planteándose un síndrome nefrótico secundario (postinfeccioso). Se trató con prednisona sin obtener remisión.

Se realizó biopsia renal que confirmó una GEFS. Continuó con proteinuria masiva, recibió tratamiento durante el año siguiente con esteroides, enalapril, losartan y ciclofosfamida, sin respuesta. Progresa hacia IRC y a los 7 años de edad se constató creatinina plasmática de $4,7 \mathrm{mg} / \mathrm{dl}$, con una depuración de creatinina de $15 \mathrm{ml} / \mathrm{min}$ por cual se inició peritoneodiálisis. Se solicito estudio genético y se documentó mutación en el gen de la podocina: A284V y R229Q.

\section{Discusión}

El SN idiopático engloba un grupo heterogéneo de enfermedades renales, siendo algunas de ellas de diagnóstico clínico sencillo, pero para otras se hace necesario el apoyo de métodos diagnósticos complementarios, dada la superposición de fenotipos. El curso de estas enfermedades es variable dependiendo de su etiología. Es así como algunos pacientes evolucionan con severa proteinuria como en el SN congénito y otros tienen proteinuria moderada como en la GEFS. Esta variabilidad también se observa en el compromiso de la función renal, la cual en algunos pacientes se mantiene estable a lo largo del tiempo, y otros progresan a la IRC $^{16}$.

Este síndrome clásicamente se ha dividido en $\mathrm{SN}$ corticosensible (80-90\% de los pacientes pediátricos) y SN cortico-resistente (10$20 \%$ ) dependiendo de la respuesta a la terapia con esteroides. La mayoría de los pacientes presentan una evolución favorable con el trata- 
miento, aunque algunos pueden recaer en el transcurso de los años ${ }^{15}$.

$\mathrm{Si}$ bien el SN idiopático corresponde generalmente a una enfermedad de aparición esporádica, existen casos en que la etiología involucra factores genéticos. Así, este síndrome parece ser más severo en población afro-americana y el análisis genético en modelos animales y casos familiares en humanos, han mostrado evidencia sobre el rol patogénico de alteraciones moleculares ${ }^{15}$.

De los dos casos anteriormente comentados, la historia clínica y los antecedentes familiares del primer paciente hacen sospechar una probable base genética, pero en el segundo caso era poco probable, lo cual plantea la utilidad de solicitar estudio genético a todos aquellos pacientes con SN que no respondan al tratamiento clásico.

El hallazgo de fusión de los pedicelos observado en microscopía electrónica ha sugerido un rol clave del podocito en la patogénesis del SN idiopático $^{1}$. Boute y cols, describieron una mutación autosómica recesiva de la proteína Podocina, en SN. Otras proteínas: Nefrina, CD2AP y $\alpha$-actinina- 4 , componentes de la barrera de filtración glomerular, han sido recientemente descubiertas por estudio genético y se localizan en el diafragma de la hendidura de filtración ${ }^{15,17}$ (figura 1).

La Nefrina es una proteína transmembrana de 1241 aminoácidos perteneciente a la superfamilia de las inmunoglobulinas, codificada por el gen NPHS 1 localizado en el cromosoma 19q13. Presenta un $82 \%$ de homología con la nefrina de rata y se ha reportado su expresión en tejido nervioso, testículos y páncreas. Actuaría como molécula señal, cuyos residuos de tirosina en el dominio intracelular al ser fosforilados activan una cascada de señales antiapoptóticas $^{16,18,19}$. Las más de 70 mutaciones descritas para su gen se asocian al SN congénito tipo Finés, existiendo distintos fenotipos en cuanto a gravedad del cuadro ${ }^{20}$. CD2AP es una proteína intracelular que une el sitio citoplasmático $\mathrm{CD} 2$ a proteínas de membrana celular de células $\mathrm{T}$ y natural killer. Interactúa con nefrina y la ancla al citoesqueleto. Se ha determinado que ratones sin esta proteína presentan SN y falla renal. Kim y cols (2003), describieron una mutación en uno de los alelos en 2 pacientes con GEFS ${ }^{19,20}$.

Por otra parte, $\alpha$-Actinina-4 corresponde a un tipo de filamento de actina que asegura la integridad morfológica del podocito, codificada por el gen ACTN4, cuya alteración molecular se ha observado en GEFS autosómico dominante $^{20}$.

La Podocina es una proteína integral de membrana de 42-kda y 383 aminoácidos, ubicada en la membrana del diafragma de la hendidura de filtración, codificada por el gen NPHS2, ubicado en el cromosoma 1q25-31. Su mutación se ha asociado al SN cortico-resistente autosómico recesivo ${ }^{18}$, que se caracteriza por un desarrollo precoz de la enfermedad (menor de 6 años), con proteinuria, progresión a enfermedad renal crónica y cambios histológicos

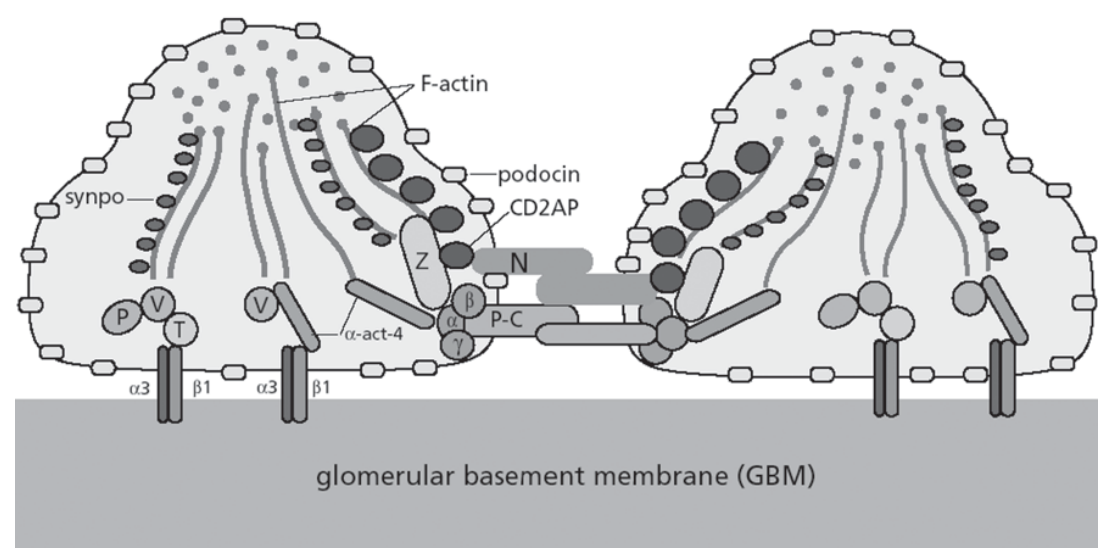

glomerular basement membrane (GBM)
Figura 1. La figura muestra esquemáticamente la unión entre los pedicelos y las proteínas constituyentes de la hendidura de filtración, nefrina, podocina, CD2AP y el filamento de anclaje al citoesqueleto, $\alpha$-actinina- 4 . En Nature genetics 2000; 24: 333-5. 
compatibles con GEFS o nefropatía por cambios mínimos ${ }^{21}$. Hasta la fecha, se han descrito más de 30 mutaciones, afectando principalmente el dominio C-terminal. La Podocina interactúa con las proteínas Nefrina y CD2AP, cumpliendo una función estructural, estabilizando las interacciones entre estas proteínas y anclándolas a los filamentos de actina del citoesqueleto de los pedicelos ${ }^{1,18,21}$.

En niños con GEFS han sido identificadas mutaciones en el gen NPHS2. Tsukaguchi et al, publicó un estudio de 30 familias con GEFS y herencia aparentemente recesiva y 91 pacientes con GEFS primaria, en los cuales se buscó alguna mutación en dicho gen. En 9 de las 30 familias se logró demostrar una mutación, 6 de las cuales presentaron una sustitución heterocigota en el aminoácido R229Q. La frecuencia de la variación del alelo fue de un 3,6\% en la población control. Purificando las proteínas Podocina y Nefrina in vitro se estudió el efecto de esta mutación, revelando una disminución en la adhesión de ambas proteínas ${ }^{22}$.

Las mutaciones R229Q únicas, parecen ser insuficientes para causar GEFS, pero aumentan la susceptibilidad a la injuria renal en estado heterocigoto, en asociación a una segunda mutación en los alelos de NPHS $2^{22}$. Otra mutación recientemente identificada es $\mathrm{A} 284 \mathrm{~V}$, en la que se produce la sustitución de alanina por valina en la posición 851 , cuyos efectos aún se encuentran en fase experimental ${ }^{21}$. Las dos mutaciones señaladas fueron detectadas en nuestros dos pacientes, cuyo porcentaje de certeza es del $100 \%$, ya que durante el procesamiento de las muestras se realizan contrapruebas tanto para controles positivos y negativos.

\section{Conclusión}

El curso del SN está determinado en gran medida por la respuesta a corticoides, respuesta que se relaciona a la forma histológica, $y$ desde el punto de vista clínico, a la evolución y pronóstico de la enfermedad. En los pacientes con SNCR se hace necesario enfrentar largos y complicados tratamientos en base a drogas inmunosupresoras, con efectos colaterales y reacciones adversas a medicamento que deben ser permanentemente monitoreadas. En los pacientes con GEFS un 50\% evoluciona a insuficiencia renal crónica y un $25 \%$ reproduce la enfermedad en el riñón trasplantado ${ }^{13}$. De esta manera, la identificación de estas mutaciones tiene importancia clínica al diferenciar el grupo de pacientes que deberían responder a la terapia y cuales no, e igualmente a la evolución y manejo post trasplante renal en los pacientes que finalmente lo requieran. Por esta razón, el estudio genético en $\mathrm{SN}$, debería ser solicitado toda vez que sea posible. Es de esperar que con el transcurso de los años dispongamos de mejor y mayor evidencia que permita instaurar de manera masiva el estudio genético en $\mathrm{SN}$, para mejorar el tratamiento y pronóstico de este grupo de pacientes.

\section{Referencias}

1.- Arneil GC: The nephrotic syndrome. Pediatr Clin North Am 1971; 18: 547-91

2.- Chesney $R$ : The changing face of childhood nephritic syndrome. Nephrology forum. Kidney international, 2004; 66: 1294-302.

3.- Gordillo P, García A, Mota H: Síndrome nefrótico ideopático. Rev Mex Pediatr 1970; 4: 185-98.

4.- Hodson E: The management of idiopathic nephrotic syndrome in children. Paediatr Drugs 2003; 5: 33549.

5.- Hodson E: Corticosteroid therapy for nephrotic syndrome in children. Cochrane Database Syst Rev 2000: CD001533.

6.- Eddy A, Symons J: Nephrotic syndrome in childhood. Lancet 2003; 362: 629-39.

7.- Niaudet P: Genetic forms of nephrotic syndrome. Pediatr Nephrol 2004; 19: 1313-8.

8.- Mcbryde K, Kershaw D, Smoyer W: Pediatric steroidResistant Nephrotic syndrome. Curr Probl Pediatr 2001; 31: 275-307.

9.- Castaño I, Bolaños L: Colombia Medica 2005, Volumen 36 número.

10.- Latta K, Von Schnakenburg C, Ehrich J: A metaanalysis of cytotoxic treatment for frequently relapsing nephrotic syndrome in children. Pediatr Nephrol 2001; 16: 271-82.

11.- Durkan A: Effect of cytotoxic drugs in frequently relapsing nephrotic syndrome with and without steroid dependence. N Engl J Med 1982; 306: 451-4.

12.- Vogel A, Azócar M, Nazal V, Salas P: Actualizaciones en el tratamiento del Síndrome Nefrótico Idiopático Recomendaciones de la Rama de Nefrología de la Sociedad Chilena de Pediatría. Rev Chil Pediatr 2006; 77 (3): 295-303.

13.-Durkan AM: Non-corticosteroid treatment for 
nephrotic syndrome in children. Cochrane Database Syst Rev 2005: CD002290.

14.- Niaudet $P$, Broyer $M$, Habib R: Treatment of idiopathic nephrotic syndrome with cyclosporin A in children. Clin Nephrol 1991; 35: 31-6.

15.- Antignac $C$ : Genetic models: clues for understanding the pathogenesis of idiopathic nephrotic syndrome. J Clin Invest 2002; 109: 447-9.

16.- Tryggvason K, Patrakka J, Wartiovaara: Hereditary Proteinuria Syndromes and Mechanisms of Proteinuria. N Engl J Med 2006; 354: 1387-401.

17.- Somlo S, Mundel P: Getting a foothold in nephrotic syndrome. Nat Genet 2000; 24: 333-5.

18.- Aaltonen P, Holthöfer $H$ : Nephrin and related proteins in the pathogenesis of nephropathy. Drug Discov
Today: Disease Mechanisms 2007; 4: 21-7.

19.- Patrakka J, Tryggvason $K$ : Nephrin- $\alpha$ unique structural and signaling protein of kidney filter. Trends in Molec Med 2007; 13 (9): 396-403.

20.- Kawachi H, Miyaauchi N, Suzuki K, Han G, Orikasa M, Shimizu F: Role of podocyte slit diaphragm as a filtration barrier. Nephrology 2006; 11: 274-81.

21.- Karle S, Uetz B, Ronner V, Glaeser L, Hildebrandt F, Fuchshuber A: Novel Mutations in NPHS2 detected in both familial and sporadic steroid-resistant nephritic syndrome. J Am Soc Nephrol 2002; 13: 388-93

22.- Tsukaguchi H, Sudhakar A, Cam Le T, et al: NPHS2 mutations in late-onset focal segmental glomerulosclerosis: R229Q is a common disease-associated allele. J Clin Invest 2002; 110 (11): 1659-66. 